\title{
ARTÍCULOS
}

Sometido 22.07.2016. Aprobado 19.06.2017

Evaluado por el sistema double blind review. Editora Científica: Fernanda Finotti Perobelli

DOI: http://dx.doi.org/10.1590/So034-759020170402

\section{REIT EN BRASIL: UNA OPORTUNIDAD DE DIVERSIFICACIÓN INTERNACIONAL}

\author{
REITs brasileiros: Uma oportunidade de diversificação internacional \\ REIT in Brazil: An opportunity of international diversification
}

MARIA CELIA LÓPEZ-PENABAD

celia.lopez@usc.es

Profesora de la Universidad de Santiago de Compostela, Facultad de Ciencias

Económicas y Empresariales - Santiago de Compostela Galicia, España

\section{CARMEN LÓPEZ-ANDIÓN}

carmen.lopez.andion@usc.es Profesora de la Universidad de Santiago de Compostela, Facultad de Ciencias Económicas y Empresariales - Santiago de Compostela Galicia, España

\section{ANA IGLESIAS-CASAL \\ ana.iglesias.casal@usc.es Profesora de la Universidad de Santiago de Compostela, Facultad de Ciencias Económicas y Empresariales - Santiago de Compostela - Galicia, España}

\section{JOSÉ MANUEL MASIDE-SANFIZ}

josemanuel.maside@usc.es Profesor de la Universidad de Santiago de Compostela, Facultad de Ciencias

Económicas y Empresariales - Santiago de Compostela Galicia, España

\begin{abstract}
RESUMEN
Los beneficios de la diversificación internacional se han visto modificados por el cambio continuo de las correlaciones entre los mercados. La inversión inmobiliaria presenta un elevado potencial como activo diversificador en un portafolio. El objetivo del presente trabajo es analizar la diversificación combinando la inversión en REIT con renta variable, para un inversor de Brasil. Para ello construimos carteras óptimas out-of-sample mediante predicciones de volatilidad, correlaciones y rendimientos utilizando los métodos DCC y VAR-DCC para el índice Ifix y los ETF BOVA11 y SPDR S\&P500, durante el período de 1 de diciembre de 2014 a 30 de octubre de 2015. Nuestros resultados muestran que la aproximación VAR-DCC mejora ligeramente las aproximaciones Plug-in y DCC, y la cartera Naïve.
\end{abstract}

PALABRAS CLAVE | Real Estate Investment Trust, diversificación internacional, Modelo VAR-DCC multivariante, Brasil, Ratio de Sharpe.

\section{RESUMO}

Os benefícios da diversificação internacional estão sendo modificados pela mudança contínua das correlações entre os mercados. $O$ investimento imobiliário apresenta um elevado potencial enquanto ativo diversificador de uma carteira. $O$ objetivo do presente trabalho é analisar a diversificação mediante $o$ investimento em REITs e renda variável, para um investidor no Brasil. Então nós construímos carteiras ótimas out-of-sample mediante previsões da volatilidade, correlações e rendimentos utilizando os modelos (métodos) DCC e VAR-DCC para o índice Ifix, e os ETFS BOVA11 e SPDR S\&P500, durante o período de 1 de dezembro de 2014 a 30 de outubro de 2015. Os nossos resultados mostram que a aproximação VAR-DCC melhora a aproximação Plug-in e a DCC, e a estratégia Naïve.

PALAVRAS-CHAVE / Real Estate Investment Trust, Diversificação internacional, Modelo multivariado VAR-DCC, Brasil, Rácio de Sharpe.

\section{ABSTRACT}

The benefits of international diversification have been modified by the continuous change in the correlations between markets. Real estate investment has a high potential as a diversifying asset in a portfolio. The aim of this paper is to analyze the diversification by investing in REITs and equities for an investor from Brazil. We build optimal portfolios out-of-sample using predictions of volatility, correlations and yields following the DCC and VAR-DCC methods for the BOVA11, Ifix and SPDR S\&P500 indices, during the period from December 1, 2014 to October 30, 2015. Our results show that the VAR-DCC approach slightly improves the DCC and Plug-in approaches, and the Naïve strategy.

KEYWORDS / Real Estate Investment Trust, international diversification, multivariate VAR-DCC model, Brazil, Sharpe Ratio. 


\section{INTRODUCCIÓN}

En el marco de los mercados financieros globales de constante cambio en las correlaciones, la inversión inmobiliaria se plantea cada vez más como una opción a nivel internacional, que en busca de rendimientos atractivos y diversificación, pone su atención en mercados emergentes como puede ser el de Brasil, siempre como parte de una inversión siguiendo un enfoque táctico.

En los últimos 20 años el estado de Brasil ha puesto todo su empeño en mejorar las condiciones jurídicas e institucionales para tratar de fortalecer los negocios con la propiedad privada del suelo y relacionarlos con el sistema financiero. De esta forma, cada vez más agentes financieros globales obtienen rendimientos a partir de la ininterrumpida reconfiguración de espacios urbanos en Brasil (Sanfelici, 2013).

Rentabilizar las inversiones en mercados emergentes es una tarea complicada. La falta de transparencia e información dificultan el proceso de inversión. Los rendimientos de este tipo de inversiones pueden venir generados por el desarrollo económico, la escasez de capital y/o el desarrollo demográfico. En el caso de Brasil, el fuerte crecimiento de su población -ingrediente fundamental para una demanda inmobiliaria intensa $y$, por tanto, para el alza del precio de este tipo de bien- genera gran interés internacional por la inversión inmobiliaria en este mercado.

La rentabilidad y liquidez son aspectos fundamentales a tener en cuenta en cualquier tipo de inversión, también en la inversión inmobiliaria, si bien su potencial como activo que diversifica el riesgo en un portafolio cobra una especial relevancia. En este tema resulta fundamental el análisis de las correlaciones existentes entre los distintos activos; si las variables fundamentales que determinan los rendimientos inmobiliarios están poco correlacionadas también lo estarán los activos.

Es importante poner de manifiesto que la inversión inmobiliaria directa presenta desventajas importantes, como la baja liquidez y los elevados costes de transacción. Sin embargo, la inversión colectiva inmobiliaria mitiga este tipo de dificultades presentando las ventajas de cualquier inversión colectiva, como por ejemplo la gestión profesionalizada, la transparencia y el control de su actividad (Milani, Ceretta, \& Machado, 2014).

El objetivo de nuestro trabajo es analizar el potencial de diversificación mediante la inversión en REIT (Real Estate Investment Trust) en el mercado de Brasil -instituciones que se han desarrollado de forma extraordinaria en los últimos añosa través de la inversión en el Ifix, índice del mercado de REIT en Brasil. Inversión que combinamos con renta variable de Brasil y EE.UU., mediante los ETF BOVA11 y SPDR S\&P500. Como indicador de la performance recurrimos a la Ratio de Sharpe. Utilizamos modelos de predicción multivariantes VAR-DCC de los rendimientos, volatilidades y correlaciones, que comparamos con los modelos tradicionales Media-varianza de Markowitz (1952) o modelo Plug-in. En el procedimiento de optimización seguimos el modelo propuesto por Kirby y Ostdiek (2012) que incorpora como restricción el rendimiento de la cartera Naïve. Como prueba de robustez utilizamos tres tipos de cópulas que permiten dependencia variando en el tiempo: normal, SJC y cópula t.

Consideramos que nuestro trabajo supone un importante valor añadido a la literatura existente por dos razones. En primer lugar, en él se analizan combinaciones de un producto relativamente nuevo, como es el de los REIT de Brasil, con la renta variable doméstica y extranjera de una economía de referencia como EE.UU., poniendo de manifiesto gran potencial de diversificación de los productos de Brasil en combinación con la renta variable estadounidense. En segundo lugar, se contribuye a la literatura sobre gestión de carteras óptimas dinámicas mediante predicciones de volatilidad y rendimientos empleando las aproximaciones DCC y DCC-VAR multivariantes y modelos de cópulas. Nuestros resultados muestran que el mejor portafolio en términos de la Ratio de Sharpe es el obtenido mediante la aproximación VAR-DCC. Para ello, en el siguiente apartado presentamos este tipo de instituciones y su desarrollo en el mercado.

\section{FONDOS DE INVERSIÓN INMOBILIARIA EN BRASIL}

Los Fondos de Inversión Inmobiliaria (FII) en Brasil, conocidos como "Fundos de Investimento Inmobiliario" fueron introducidos por primera vez mediante la Ley $\mathrm{n}-8.668$ del 25 de junio del año 1993. En esta ley se establece que serán fondos sin personalidad jurídica caracterizados por la reunión de los recursos recaudados a través del sistema de distribución de valores mobiliarios, de conformidad con la ley $n^{\circ} 6.385$ de 1976 , para la aplicación en el sector inmobiliario. Los Fll son de naturaleza cerrada y el reembolso de acciones está prohibido por un período de duración limitada o ilimitada, son gestionados por instituciones financieras y supervisados por la Comisión de Valores Mobiliarios (CVM), que es un organismo independiente vinculado al Ministerio de Economía en Brasil, encargado de supervisar los mercados de valores, velar por la transparencia, la correcta formación de precios y la protección de los inversores. 
La estructura de los Fll se conforma por la entidad gestora, la política de inversión, el precio de entrada, la remuneración del administrador, los poderes y quórum de votación, y el período de reembolso, entre otros.

En el año 1999, por medio de la ley nº 9.779 se establecen cambios en la legislación fiscal sobre la renta, atribuida a los FII y los ingresos obtenidos (fijos o variables). En esta ley se dicta que el fondo deberá distribuir entre sus accionistas al menos el $95 \%$ de los beneficios obtenidos, calculado sobre una base de efectivo, basado en la declaración o balance trimestral terminado el 30 de junio y el 31 de diciembre de cada año.

En la actualidad, están regulados por la Instrucción 472 de la CVM del año 2008 que prevé la creación, gestión, funcionamiento, oferta pública de acciones y divulgación de los FII. Los FIl en Brasil, tanto por sus características como por su funcionamiento, se engloban en la categoría de REIT.

La propia normativa del mercado de FII en Brasil señala como ventajas de la inversión en estas entidades: la accesibilidad, la diversificación, la aplicación, flexibilidad y transparencia, entre otros. Por otro lado, también destaca la existencia de riesgos en las inversiones que dependen de la naturaleza y los objetivos del fondo, así como de las crisis políticas y económicas, tanto nacionales como internacionales.
Este tipo de inversión, la inversión colectiva inmobiliaria, permite a cualquier persona realizar una inversión diversificada en bienes raíces (incluyendo apartamentos, hospitales, hoteles, instalaciones industriales, infraestructuras, oficinas, centros comerciales, centros de almacenamiento y bosques maderables, entre otros), mediante la adquisición de valores o participaciones, ofreciendo ingresos derivados del alquiler o venta de las propiedades que se poseen a largo plazo.

Brasil presenta una industria de FII desarrollada y de gran tamaño. A pesar de ello, se pueden considerar un instrumento de inversión relativamente nuevo ya que, aunque fueron regulados en 1993, su desarrollo no tuvo lugar hasta el año 2003, año en que empezaron a cotizar en bolsa los primeros FIl.

De acuerdo con la CVM, en febrero de 2015 se encontraban registrados aproximadamente 12.800 fondos de inversión, con un patrimonio líquido de $\mathrm{R} \$ 2,47$ billones. Del total de fondos de inversión en Brasil en el año 2015, el 2,04\% son FII (386 en marzo de 2015).

En el siguiente gráfico, se muestra la evolución del número de fondos de inversión entre los años 2009-2015 y el número de fondos que cotizan en la BM\&FBOVESPA del total registrados en la CVM.

\section{Gráfico 1. Evolución del número de FII registrado en la CVM en Brasil entre 2009-marzo 2015}

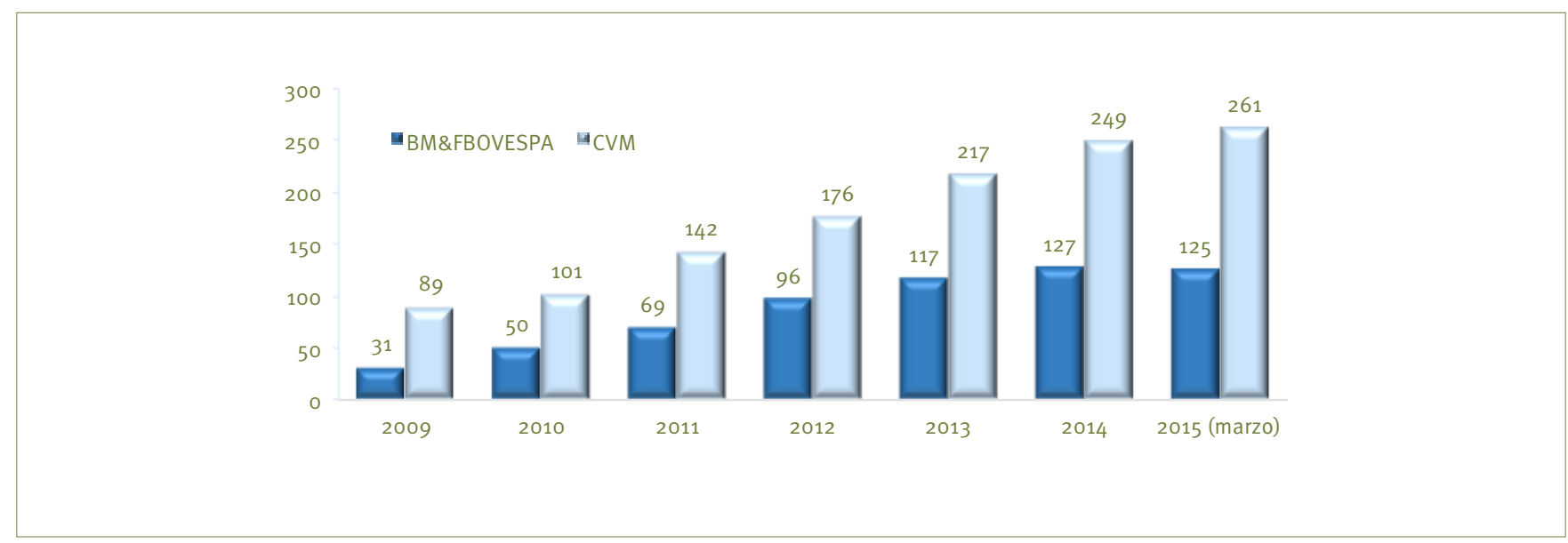

Fuente: Datos del Informe de mercado de la BM\&FBovespa (2015).

Como se puede apreciar en el Gráfico 1 anterior, el número de FII a lo largo de los años ha venido incrementándose, pasando de 89 a 261 fondos. Igualmente, se puede observar que se ha incrementado el número de fondos que cotizan en la BOVESPA, pasando de 31 fondos a 125. En marzo de 2015, el 48\% del total de Fll estaban cotizando.

A continuación, en el Gráfico 2, se muestra la evolución del patrimonio líquido y el valor de mercado de los Fll para el período comprendido entre abril de 2013 y marzo de 2015. Se puede observar que el patrimonio líquido tiene un comportamiento ascendente y valor de mercado descendente. Esto nos indica que, en los últimos años, los inversores han sufrido una bajada en los valores de 
mercado de los FII mientras que su patrimonio mantiene una ligera tendencia alcista. Este distanciamiento entre las dos magnitudes puede deberse a la desaceleración económica, a la fuga de inversores debido a los riesgos de liquidez, a la caída de los precios de las acciones y, por otro lado, al hecho de que el real brasileño se ha visto debilitado frente al dólar en un 20\% desde el año 2014 .

Gráfico 2. Evolución del patrimonio líquido y el valor de mercado de FIl en Brasil (2013-2015)

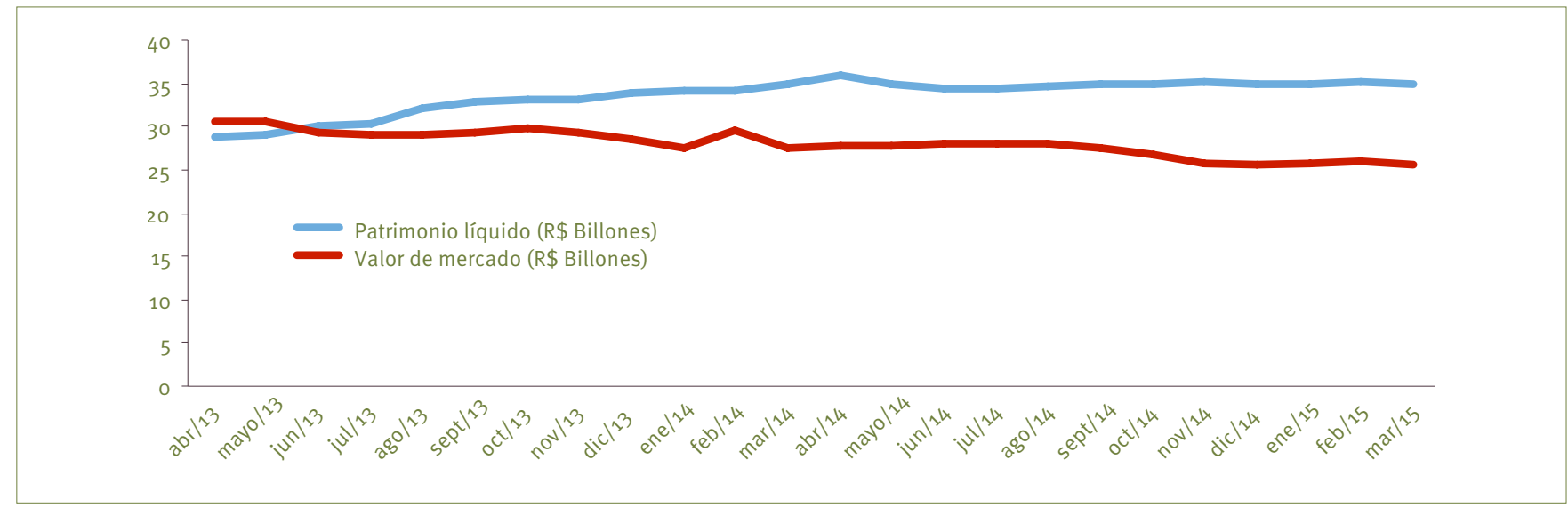

Fuente: Elaboración propia, con datos del Informe de mercado de la BM\&FBovespa (2015).

Analizando el número de inversores en los Fll de Brasil se aprecia una disminución de los mismos en un período de un año (marzo 2014-marzo 2015) del 6\%. En el siguiente Gráfico 3 se muestra el comportamiento del número de inversores a lo largo del último año.

Gráfico 3. Número de inversores en Brasil entre marzo 2014-marzo 2015

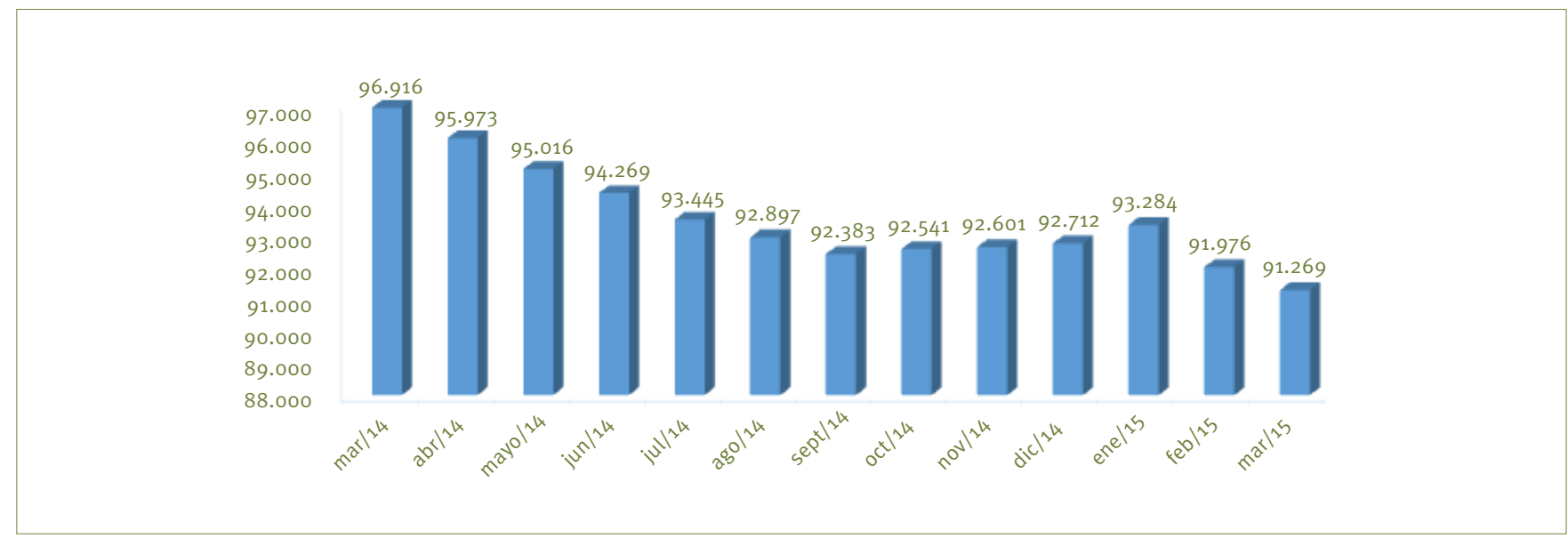

Fuente: Datos del Informe de mercado de la BM\&FBovespa (2015).

El número de Fll ha ido aumentando en los últimos años pero, al mismo tiempo, disminuyó su valor en el mercado y el número de inversores. Este comportamiento derivó de la difícil situación política y económica que afrontó Brasil y del escándalo producido por Petrobras y algunas de las empresas de construcción e ingeniería más importantes, que generó desconfianza en los inversores extranjeros y nacionales.

Existen diferentes segmentos de inversión para los Fll de Brasil en los cuales se enmarcan los Fll que cotizan en la BM\&FBOVESPA. En el siguiente Gráfico 4 se puede observar la distribución de los fondos por segmento, donde el 35\% de los fondos se concentra 
en la inversión de edificaciones para oficinas; el 16\%, en el segmento de centros comerciales y el 14\%, en el de cuentas por cobrar. El segmento menos representativo es el de hotelería, con $1 \%$.

\section{Gráfico 4. Número de fondos por segmento de inversión en Brasil}

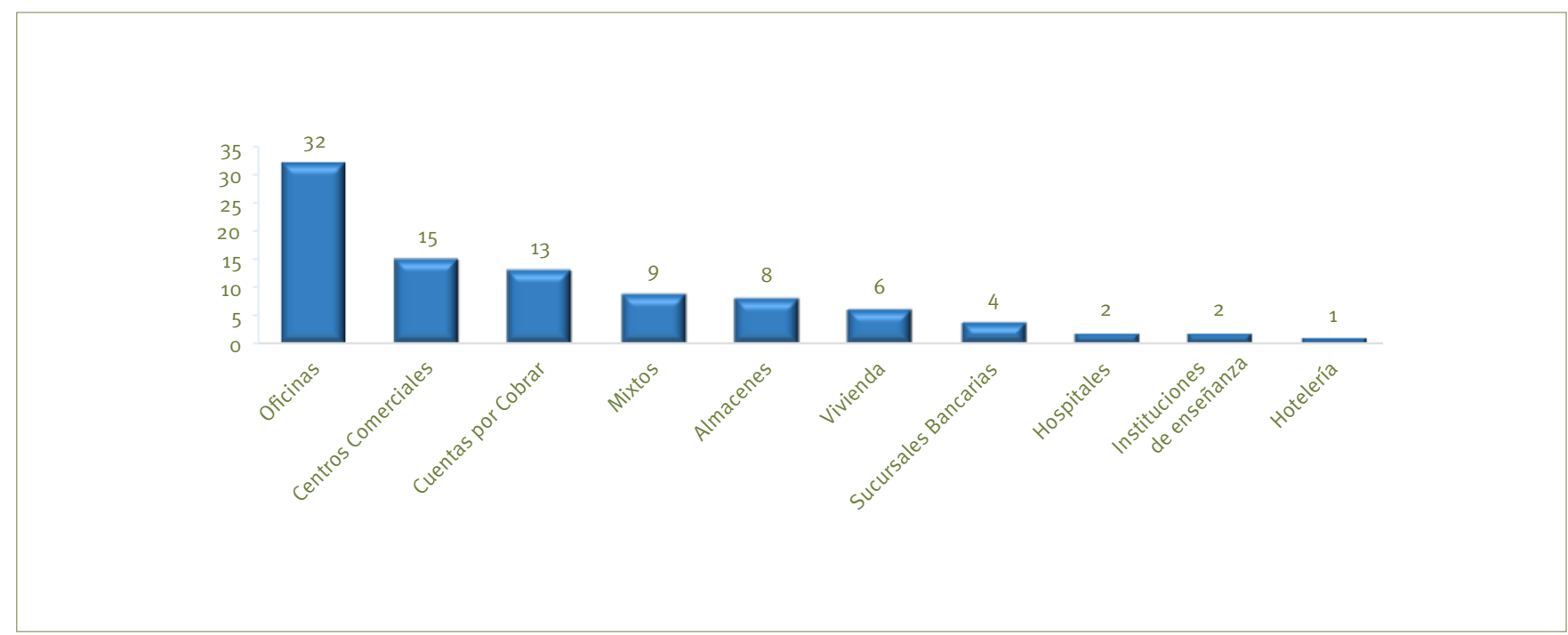

Fuente: Datos de InfoMoney (2014).

Además, existen trece fondos que cotizan en la bolsa que se dedican a la adquisición de cuotas de otros Fll, Certificados de Cuentas Inmobiliarias (CRI), Letras de Crédito Inmobiliario (LCI), Letras de Hipotecas (LH) y Títulos del Tesoro Nacional.

Es importante destacar que este tipo de fondos inmobiliarios suponen un riesgo importante, al ser un mercado relativamente nuevo, los inversores tienen dudas sobre las expectativas de rentabilidad. Además, las inversiones en países emergentes siempre son más arriesgadas, especialmente cuando el país en cuestión atraviesa circunstancias complicadas como las vividas en Brasil.

Una característica importante de los REIT -que los hace especialmente atractivos para los inversores internacionales- es su exención fiscal en el impuesto de sociedades, que permite que los inversores eviten la doble imposición internacional que tienen la mayoría de las acciones. Sin embargo los REIT ofrecen menos diversificación que la inversión inmobiliaria privada y que los fondos que no cotizan en mercados bursátiles. Los rendimientos de los REIT tienden a estar más correlacionados con la renta variable y fija. Por lo tanto, los REIT son ideales para pequeños inversores no especializados que quieren mantener cierta exposición a la inversión inmobiliaria en su cartera, y también para inversores institucionales que necesitan liquidez.

\section{METODOLOGÍA Y DATOS}

\section{METOdología}

Tal y como hemos comentado anteriormente, el objetivo de nuestro trabajo es estudiar el potencial de la inversión colectiva inmobiliaria como activo para lograr una diversificación eficiente en una cartera mediante la utilización de modelos multivariantes. Es decir, analizaremos la performance para un inversor de Brasil que construye portafolios óptimos mediante predicciones de volatilidad, correlaciones y rendimientos con modelos multivariantes para el índice Ifix, y los ETF BOVA11 y SPDR S\&P500.

En primer lugar, presentamos la metodología de modelos multivariantes DCC utilizados para estimar los rendimientos, las varianzas y correlaciones condicionales para las 3 inversiones (Ifix, BOVA11 y SPDR S\&P500). En segundo lugar, detallamos la estrategia de elección de portafolio óptimo que nos permita una diversificación internacional. Por último, definimos el indicador de evaluación de la performance de las estrategias alternativas y el método de inferencia utilizado para valorar la significación estadística de las diferencias de la performance entre cada alternativa y la cartera considerada como benchmark.

Nuestro planteamiento es utilizar la estrategia Naïve como nuestro benchmark, pero no como propuesta de estrategia a 
seguir. Es frecuente la inclusión de la estrategia Naïve como referente ya que es muy fácil de aplicar, no necesita ninguna estimación previa ni tampoco ser optimizada y los inversores continúan utilizando esta simple regla de asignación de activos; además, resulta bastante eficiente cuando el número de activos del portafolio no es reducido.

\section{Análisis multivariante}

Un modelo GARCH multivariante general está definido como:

$$
\begin{aligned}
& Y_{t}=\mu_{t}+\varepsilon_{t} \\
& \varepsilon_{t}=H_{t}^{1 / 2} v_{t} \\
& H_{t}=D_{t} R_{t} D_{t}
\end{aligned}
$$

donde $Y_{t}$ es un vector de orden nxi de rendimientos, $\mu_{t}$ es un vector nx1 de la esperanza condicional de $Y_{t}$, que contiene, siguiendo los resultados obtenidos en el análisis univariante, constantes o cualquier proceso de series de tiempo como un ARMA(P,Q) o un VAR(k), $H_{t}$ es la matriz de covarianzas condicionales de las perturbaciones $\varepsilon_{t} ; v_{t}$ es un vector de variables aleatorias idéntica e independientemente distribuidas (i.i.d.) de media cero $E\left[\vartheta_{t}\right]=0$ y varianza unitaria $E\left[\vartheta_{t} \vartheta_{t}^{\prime}\right]=I, \mathrm{D}_{\mathrm{t}}$ es una matriz diagonal de desviaciones condicionales en la cual sus elementos se definen mediante un proceso t-TARCH(p,q) univariante como:

$$
\sigma_{i, t}^{2}=w_{i}+\sum_{j=1}^{p} \alpha_{i} \varepsilon_{i, t-j}^{2}+\lambda_{1} d_{t-1} \varepsilon_{i, t-1}^{2}+\sum_{j=1}^{q} \beta_{i} \sigma_{i, t-j}^{2}
$$

donde $d_{t-1}$ es una variable ficticia que es igual a uno si $\varepsilon_{t-1}<0$ y cero si $\varepsilon_{t-1} \geq 0 ; \lambda_{1}$ captura el efecto apalancamiento y se considera que $\varepsilon_{t}$ sigue una distribución $t$ de Student con u grados de libertad. En el modelo DCC propuesto por Engle (2002) la matriz de correlaciones condicionales $R_{t}$ se define como:

$$
R_{t}=\left(\operatorname{diag}\left(Q_{t}\right)\right)^{-1 / 2} Q_{t}\left(\operatorname{diag}\left(Q_{t}\right)\right)^{-1 / 2}
$$

siendo:

$$
Q_{t}=\left(1-\theta_{1}-\theta_{2}\right) \bar{Q}+\theta_{1} z_{t-1} z_{t-1}^{\prime}+\theta_{2} Q_{t-1}
$$

en la que $\overline{\mathrm{Q}}$ es la mātriz de covarianzas incondicionales de los residuos estandarizados, $z_{t}$. Además, en un modelo DCC los parámetros $\theta_{1}$ y $\theta_{2}$ son escalares idénticos para todas las correlaciones condicionales.

La implementación de estos modelos requiere la especificación previa de la media y varianza condicional de cada rendimiento y la posterior estimación del proceso univariante. Luego las observaciones se estandarizan utilizando esas varianzas condicionales estimadas y se obtiene la matriz de covarianzas incondicionales. En una segunda etapa se maximiza la siguiente función:

$$
L_{2 T}(\theta)=-\frac{1}{2} \sum_{t=1}^{T}\left(n \log (2 \pi)+2 \log \left|D_{t}\right|\right)-\frac{1}{2} \sum_{t=1}^{T} \log \left|R_{t}\right|-\frac{1}{2} \sum_{t=1}^{T} z_{t}^{\prime} R_{t}^{-1} z_{t}
$$

lo que nos permite estimar los parámetros $\theta_{1}$ y $\theta_{2}$, y en consecuencia las correlaciones condicionales. 


\section{Elección de la cartera óptima}

Utilizaremos la teoría de la elección de la cartera óptima propuesta por Markowitz (1952) que consiste en minimizar el riesgo sujeto a la restricción de que el rendimiento esperado del portafolio sea como mínimo el de la cartera de referencia, $R^{\star}$. Siguiendo a Kirby y Ostdiek (2012), $R^{\star}$ es el rendimiento esperado de la estrategia Naïve, que consiste en invertir en cada activo la misma proporción, $1 / \mathrm{N}$ en el caso de $\mathrm{N}$ activos. Estos autores demuestran que si el modelo Media-varianza se aplica estableciendo como condición que el rendimiento esperado coincida con el de la cartera equiponderada, el portafolio resultante mejorará la cartera Naïve. El planteamiento utilizado es el siguiente:

\begin{tabular}{|l|l|}
$\min w_{t}^{\prime} H_{t+1 / t} w_{t}$ & \\
$\quad$ s.a. & \\
$w_{t}^{\prime} E\left\{R_{t+1}\right\} \geq R^{*}$ & \\
$w^{\prime} 1=1$ & $(6,2)$ \\
\hline
\end{tabular}

$w_{t}$ es la matriz de proporciones, $H_{t+1}$ la de covarianzas y $E\left\{R_{t+1}\right\}$ la de rendimientos esperados.

En este caso consideramos la posibilidad de realizar ventas al descubierto y no contemplamos la posibilidad de invertir en un activo sin riesgo.

Para calcular la cartera óptima utilizamos las predicciones a un día de rendimientos y matriz de varianzas-covarianzas obtenidos con tres modelos distintos: el muestral (método Plug-in o aproximación clásica Media-varianza) que consiste en utilizar como predicción la media y la matriz de covarianzas muestrales de los rendimientos hasta el día $t$; el modelo DCC que incluye la modelización ARMA de la media condicional y el modelo VAR-DCC que modeliza la media condicional mediante un $\operatorname{VAR}(k)$. Estos dos últimos, en los que se predicen varianzas y covarianzas condicionales, presentan como ventaja la consideración de correlaciones dinámicas entre los rendimientos previstos.

\section{Evaluación de la performance}

Como indicador de performance utilizamos la Ratio de Sharpe (RS), que se define como el cociente del exceso de rendimiento esperado de una inversión con relación a su desviación estándar.
Esta elección está motivada por el hecho de que la RS es la medida ajustada por riesgo más utilizada en la práctica de los mercados financieros para evaluar la gestión y el atractivo de las distintas estrategias de inversión.

La RS en su formulación tradicional también presenta limitaciones. Básicamente, la RS es una medida que intenta calcular la recompensa (exceso de rendimiento) por unidad de riesgo (desviación estándar) de una inversión. Cuando los excesos de rendimiento son positivos, una RS mayor indica una mayor performance originada por un exceso de rendimiento mayor o una desviación típica menor, o ambos. Sin embargo si los excesos de rendimientos son negativos, la RS puede dar lugar a una ordenación equivocada. Por ejemplo, dos portafolios que alcanzan el mismo exceso de rendimiento negativo, pero con diferentes desviaciones estándar. En este caso, la RS tradicional indicaría que la cartera con la desviación estándar mayor es la mejor, a pesar de que lograría el mismo rendimiento negativo con un mayor riesgo. Israelsen $(2003,2005)$ propone una modificación simple a la RS que soluciona este problema generando una ordenación adecuada de las inversiones con excesos de rendimiento negativos. La RS modificada tiene la siguiente expresión:

$$
R S m=\left\{\begin{array}{l}
\bar{r} / \sqrt{\sigma^{2}}, \text { si } \bar{r} \geq 0 \\
\bar{r} \times \sqrt{\sigma^{2}}, \text { si } \bar{r}<0
\end{array}\right.
$$

donde $\bar{r}$ es el exceso de rendimiento, la diferencia entre el rendimiento medio de la inversión a evaluar y el activo sin riesgo; y $\sigma^{2}$ la varianza de la inversión. La RS modificada es totalmente consistente con el principio de finanzas del binomio rendimiento-riesgo, por el cual un mayor riesgo sólo es asumible a cambio de un mayor rendimiento. Así, para dos inversiones con la diferencia negativa resultará con mayor ratio aquella que tenga menor varianza.

Cuando el exceso de rendimiento es negativo, utilizamos la RS modificada para evaluar la performance de las carteras resultantes de las distintas estrategias encaminadas a estudiar el efecto diversificación de la inversión inmobiliaria.

Para valorar la significatividad de la diferencia entre la performance de la estrategia de referencia, estrategia Naïve, y la RS obtenida en la estrategia muestral o en los diferentes modelos multivariantes, se sigue la metodología propuesta por Ledoit y Wolf (2008). Ésta consiste en contrastar si la diferencia entre la RS de la estrategia utilizada y la RS de la cartera de referencia es cero mediante inferencia bootstrap. 


\section{DATOS}

De la base de datos Datastream Thomson Financial Services fueron recogidos los valores de cierre de cada sesión del índice Ifix y los ETF BOVA11 y SPDR S\&P500 para el período de 1 de enero de 2011 a 30 de octubre de 2015:

Índice Ifix, índice que refleja la evolución de los rendimientos de la industria de REIT o FII en Brasil, creado el 31 de diciembre de 2010, determinando este hecho el inicio del período a estudiar en nuestro trabajo.

ETF BOVA11, fondo indexado al índice Ibovespa, como proxy del mercado bursátil de Brasil en su totalidad.

SPDR S\&P500, fondo indexado al S\&P500 cuya muestra de cálculo incluye 500 grandes empresas que cotizan en las bolsas
NYSE o NASDAQ, considerado el más representativo del mercado de acciones de Estados Unidos.

Los estadísticos descriptivos de las series utilizadas en el presente trabajo se recogen en la Tabla 1. En ella se observa que las series de rendimientos de los índices son estacionarias (test ADF) y no se distribuyen normalmente (test JB). Los coeficientes de curtosis más elevados que los de una normal indican la mayor probabilidad de obtener valores extremos. Asimismo, se evidencia la existencia de agrupamientos de volatilidad al contrastar, mediante el estadístico de Ljung-Box, la significatividad de los coeficientes de autocorrelación de orden diez de los cuadrados y de los valores absolutos de dichos rendimientos, lo que indica la existencia de heterocedasticidad condicional en dichas series.

Tabla 1. Estadísticos descriptivos de los rendimientos de los índices Bova11, Ifix y SPDR S\&P500

\begin{tabular}{|c|c|c|c|c|c|c|c|c|c|c|c|}
\hline Rendimiento & $\mathrm{S}$ & K & JB & p-val & ADF & p-val & $\mathrm{Q}\left(\mathrm{r}_{\mathrm{t}}^{2}\right)$ & p-val & $\mathrm{Q}\left(\left|\mathrm{r}_{\mathrm{t}}\right|\right)$ & p-val & Obs. \\
\hline Bova11 & -0.171 & 5.418 & 252.254 & 0.000 & -33.779 & 0.000 & $174 \cdot 34$ & 0.000 & 127.25 & 0.000 & 1015 \\
\hline Ifix & -0.188 & 9.713 & 1911.967 & 0.000 & -12.136 & 0.000 & 118.48 & 0.000 & 137.64 & 0.000 & 1015 \\
\hline SPDR S\&P500 & -0.196 & 6.545 & 538.165 & 0.000 & -38.639 & 0.000 & 579.55 & 0.000 & 404.13 & 0.000 & 1015 \\
\hline
\end{tabular}

Nota: S es el coeficiente de asimetría; K es el de curtosis; JB es el test de Jarque-Bera de normalidad; ADF es el test de raíz unitaria de Dickey y Fuller Ampliado en el que los retardos de la ecuación auxiliar se determinan con el Criterio de Schwarz; Q(r $\left.{ }^{2}\right)$ y Q $\left(\mid r_{t}\right)$ son respectivamente el test de Ljung-Box para autocorrelación de orden diez de los cuadrados y los valores absolutos de los rendimientos. Los datos utilizados corresponden al período de estimación que comprende desde el 10 de enero de 2011 hasta el 28 de noviembre de 2014 .

Para reflejar la rentabilidad del activo sin riesgo, utilizamos la rentabilidad de la deuda pública del gobierno de Brasil a 9 meses, emitida en dólares disponibles en Datastream desde el 1 de diciembre de 2014 hasta el 30 de octubre de 2015, período de predicción.

\section{RESULTADOS EMPÍRICOS}

Consideramos la posición de un inversor de nacionalidad brasileña que toma posiciones en tres activos: uno que representa la inversión inmobiliaria, el índice Ifix; otro que representa al mercado de renta variable de Brasil, el BOVA11; y un tercero, el SPDR S\&P500, que representa la inversión en renta variable en el mercado de EE.UU. Ajustamos los rendimientos de este último teniendo en cuenta los cambios en la moneda para el inversor de Brasil. Consideramos también que dicho inversor puede reajustar su cartera diariamente.
Esta sección de resultados se divide en tres partes. En la primera parte se estiman para el período muestral los modelos DCC y VAR-DCC. Con este procedimiento tratamos de identificar la mejor especificación posible para cada modelo. En la ecuación de la media del modelo DCC hemos incluido un término constante en el caso del rendimiento del BOVA11, una estructura $\operatorname{ARMA}(4,2)$ para el rendimiento del Ifix y un $A R(1)$ para el rendimiento del SPDR S\&P500. En el modelo VAR-DCC, para la media condicional hemos seleccionado un VAR(4).

En ambos modelos, en la ecuación de la varianza condicional se considera un t-TARCH $(2,1)$ para el BOVA11, un t-TARCH $(1,1)$ para el Ifix y, finalmente, un t-TARCH(1,1) para el SPDR S\&P500. Para la ecuación de las correlaciones condicionales hemos utilizado un $\operatorname{GARCH}(1,1)$. El período muestral utilizado es el correspondiente al período del 10/1/2011 al 28/11/2014 (1.015 días). Los resultados de las estimaciones se muestran en las Tablas 2 y 3 
Tabla 2. Estimación del modelo DCC

\begin{tabular}{|c|c|c|c|}
\hline & RBova11 & RIfix & $\begin{array}{c}\text { RSPDR } \\
\text { S\&P500 } \\
\end{array}$ \\
\hline \multicolumn{4}{|c|}{ Media condicional } \\
\hline constante $_{i}$ & $-0,0451$ & 0,0163 & $0,0847^{\star \star \star}$ \\
\hline$R_{i t-I}$ & & & $-0,1266^{\star \star \star}$ \\
\hline$R_{i t-2}$ & & $0,4277^{\star \star \star}$ & \\
\hline$R_{i t-3}$ & & $0,0858^{\star \star \star}$ & \\
\hline$R_{i t-4}$ & & 0,0573 & \\
\hline \multicolumn{4}{|l|}{$\varepsilon_{i t-1}$} \\
\hline$\varepsilon_{i t-2}$ & & $-0,3211^{\star \star}$ & \\
\hline \multicolumn{4}{|c|}{ Varianza condicional } \\
\hline constante $_{i}$ & $0,0543^{* * *}$ & $0,0310^{\star * *}$ & $0,0534^{\star * *}$ \\
\hline$\varepsilon_{i t-1}^{2}$ & $-0,0482$ & $0,2003^{\star \star *}$ & $0,0726^{* *}$ \\
\hline$\varepsilon_{i t-1}^{2} * d_{t-1}$ & $0,1023^{\star * \star}$ & $0,1767^{\star}$ & $0,0980^{* *}$ \\
\hline$\varepsilon_{i t-2}^{2}$ & 0,0461 & & \\
\hline$\sigma_{i, t-1}^{2}$ & $0,9242^{\star \star *}$ & $0,5686^{* * *}$ & $0,8282^{* \star *}$ \\
\hline $\begin{array}{l}\text { tgrados de } \\
\text { libertad }\end{array}$ & $13,5943^{\star \star \star}$ & $4,4147^{\star \star \star}$ & $8,9079^{\star \star \star}$ \\
\hline \multicolumn{4}{|c|}{ Correlación condicional } \\
\hline$\theta_{1}$ & \multicolumn{3}{|c|}{$0,0217^{\star \star \star}$} \\
\hline$\theta_{2}$ & \multicolumn{3}{|c|}{$0,9624^{\star \star \star}$} \\
\hline Ln L & \multicolumn{3}{|c|}{3481,501} \\
\hline AIC & \multicolumn{3}{|c|}{6,876} \\
\hline \multicolumn{4}{|c|}{ Diagnosis de los residuos } \\
\hline Asimetría & $-0,0623$ & $-0,4296$ & $-0,2565$ \\
\hline Curtosis & 3,5983 & 8,8857 & 3,7657 \\
\hline JB & $15,80(0,000)$ & $\begin{array}{l}1496,31 \\
(0,000)\end{array}$ & $35,93(0,000)$ \\
\hline$Q(10)$ & $\begin{array}{l}3,7047 \\
(0,960)\end{array}$ & $9,2233(0,161)$ & $7,8983(0,544)$ \\
\hline$Q^{2}(10)$ & $14,276(0,161)$ & $3,8841(0,952)$ & $\begin{array}{l}5,6019 \\
(0,848)\end{array}$ \\
\hline
\end{tabular}

***,**,* representan los niveles de significatividad al $1 \%, 5 \%$ y $10 \%$ respectiva mente. JB es el test de Jarque-Bera de normalidad de los residuos estandarizados. Q(10) y Q2(10) son respectivamente los valores del test de Ljung-Box para autocorrelación de orden diez de los residuos estandarizados y de los residuos estandarizados al cuadrado.
Tabla 3. Estimación modelo VAR-DCC

\begin{tabular}{|c|c|c|c|}
\hline & RBova11 & RIfix & $\begin{array}{c}\text { RSPDR } \\
\text { S\&P500 }\end{array}$ \\
\hline \multicolumn{4}{|c|}{ Media condicional } \\
\hline$c_{\mathrm{i}}$ & $-0,0555$ & $0,0174^{*}$ & $0,0779^{\star \star \star}$ \\
\hline$R_{B O t-1}$ & $-0,0445$ & 0,0108 & $-0,1386^{\star \star \star}$ \\
\hline$R_{B O t-2}$ & 0,0205 & $-0,0008$ & $-0,0233$ \\
\hline$R_{B O t-3}$ & $-0,0259$ & 0,0084 & $-0,0234$ \\
\hline$R_{B O t-4}$ & $-0,0045$ & 0,0031 & $-0,0151$ \\
\hline$R_{I F t-1}$ & 0,0826 & $-0,0501$ & 0,0635 \\
\hline$R_{I F t-2}$ & 0,1195 & $0,1081^{\star \star *}$ & 0,0991 \\
\hline$R_{I F t-3}$ & 0,0023 & $0,0957^{\star * *}$ & 0,0238 \\
\hline$R_{I F t-4}$ & $-0,0182$ & $0,0902^{* \star *}$ & $-0,0677$ \\
\hline$R_{S P t-1}$ & 0,0501 & $-0,0186^{*}$ & $-0,0571^{\star}$ \\
\hline$R_{S P t_{-2}}$ & $-0,0435$ & 0,0079 & 0,0445 \\
\hline$R_{S P_{t-3}}$ & 0,0087 & $-0,0089$ & 0,0207 \\
\hline$R_{S P P_{t-4}}$ & $-0,01401$ & $-0,0024$ & $-0,0001$ \\
\hline \multicolumn{4}{|c|}{ Varianza condicional } \\
\hline$\omega_{i}$ & $0,0514^{* \star *}$ & $0,0272^{\star \star \star}$ & $0,0555^{* \star}$ \\
\hline$\varepsilon_{i t-1}^{2}$ & $-0,04557$ & $0,1936^{\star \star \star}$ & $0,0634^{\star}$ \\
\hline$\varepsilon_{i t-1}^{2} * d_{t-1}$ & $0,1005^{\star * *}$ & $0,1633^{*}$ & $0,1232^{\star \star \star}$ \\
\hline$\varepsilon_{i t-2}^{2}$ & 0,0416 & & \\
\hline$\sigma_{i, t-1}^{2}$ & $0,9289^{\star * *}$ & $0,6002^{\star \star \star}$ & $0,8201^{\star * *}$ \\
\hline $\begin{array}{l}\text { tgrados de } \\
\text { libertad }\end{array}$ & $13,3916^{* * *}$ & $4,3975^{\star \star \star}$ & $9,6500^{\star * *}$ \\
\hline \multicolumn{4}{|c|}{ Correlación condicional } \\
\hline$\theta_{1}$ & \multicolumn{3}{|c|}{$0,0258^{\star \star \star}$} \\
\hline$\theta_{2}$ & \multicolumn{3}{|c|}{$0,9509^{\star \star *}$} \\
\hline Ln L & \multicolumn{3}{|c|}{$-3453,71$} \\
\hline AIC & \multicolumn{3}{|c|}{6,8219} \\
\hline \multicolumn{4}{|c|}{ Diagnosis de los residuos } \\
\hline Asimetría & $-0,0564$ & $-0,3811$ & $-0,2589$ \\
\hline Curtosis & 3,6322 & 8,6292 & 3,6871 \\
\hline$J B$ & $17,44(0,000)$ & $\begin{array}{l}1364,75 \\
(0,000)\end{array}$ & $31,31(0,000)$ \\
\hline$Q(10)$ & $\begin{array}{l}3,4567 \\
(0,969)\end{array}$ & $14,791(0,140)$ & $5,320(0,869)$ \\
\hline$Q^{2}(10)$ & $15,665(0,110)$ & $4,283(0,934)$ & $5,608(0,847)$ \\
\hline
\end{tabular}

***, ${ }^{* *},{ }^{*}$ representan los niveles de significatividad al $1 \%, 5 \%$ y $10 \%$ respectiva mente. JB es el test de Jarque-Bera de normalidad de los residuos estandarizados. Q(10) y Q2(10) son respectivamente los valores del test de Ljung-Box para autocorrelación de orden diez de los residuos estandarizados y de los residuos estandarizados al cuadrado. 
Los resultados del modelo DCC (Tabla 2) muestran que la mayor parte de los coeficientes de las ecuaciones de la media y la varianza son significativos, lo que confirma la necesidad de la modelización GARCH. Los coeficientes de la ecuación de las correlaciones son altamente significativos, lo que justifica la especificación dinámica de las correlaciones, y su suma es inferior a uno por lo que esas correlaciones revierten a la media. En la última parte de la tabla se muestran las propiedades de los residuos estandarizados de cada una de las ecuaciones de rendimientos. El estadístico Q de LjungBox para el retardo de orden 10 indica ausencia de autocorrelación en los residuos al nivel de significación del $5 \%$. Este estadístico, aplicado al cuadrado de los residuos estandarizados $\left(\mathrm{Q}^{2}\right)$, indica ausencia de heterocedasticidad condicional.

En el caso del VAR-DCC (Tabla 3), el orden adecuado del VAR, 4, se ha seleccionado mediante el estadístico AIC. Los resultados obtenidos para las ecuaciones de la varianza y las correlaciones condicionales son muy similares a los del modelo anterior. Pero consideramos esta modelización más satisfactoria, teniendo en cuenta los valores del logaritmo de la función de verosimilitud y del AIC.

Gráfico 5. Correlaciones condicionales dinámicas de los rendimientos. DCC-VAR
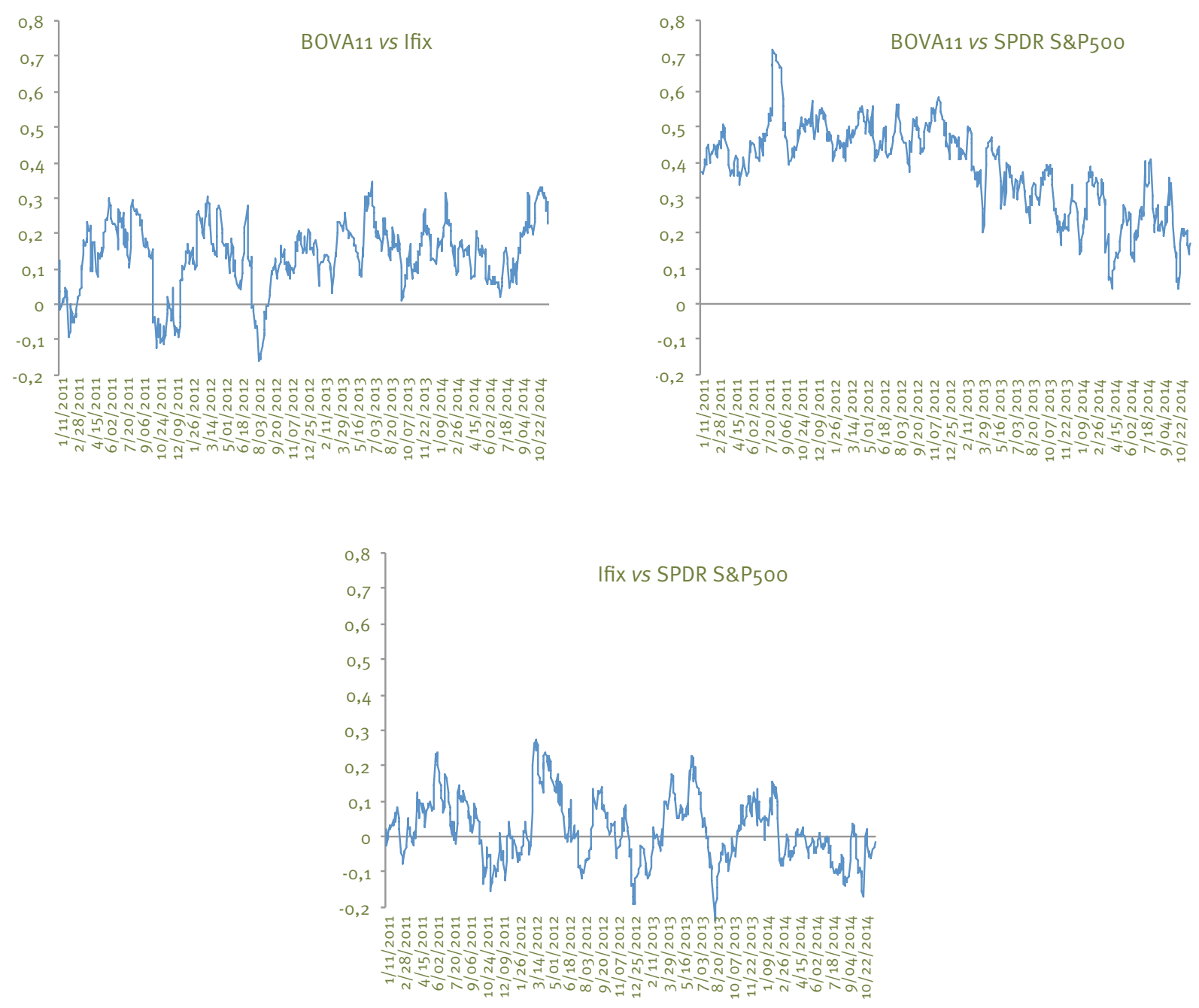
Tabla 4. Estadísticos descriptivos de las correlaciones condicionales dinámicas de los tres activos de la cartera. VAR-DCC

\begin{tabular}{l|c|c|c|c|c|c}
\hline Rendimientos & № obs. & Media & $\begin{array}{c}\text { Desv. } \\
\text { Estándar }\end{array}$ & Min. & Max. & Asimetría \\
\hline BOVA11, Ifix & 1014 & 0,137 & 0,097 & $-0,159$ & 0,348 & $-0,619$ \\
\hline $\begin{array}{l}\text { BOVA11, } \\
\text { S\&P500 }\end{array}$ & 1014 & 0,385 & 0,124 & 0,042 & 0,723 & $-0,325$ \\
\hline Ifix, S\&P500 & 1014 & 0,018 & 0,089 & $-0,238$ & 0,274 & $-0,301$ \\
\hline
\end{tabular}

En el gráfico 5 se muestra la evolución de las correlaciones dinámicas de los diferentes rendimientos obtenidas mediante el VAR-DCC. Los estadísticos descriptivos de dichas series se presentan en la tabla 4. Entre los rendimientos de BOVA11 e Ifix se observan bajas correlaciones ( 0.137 en media) alcanzando un máximo de 0.348 y un mínimo de -0.159. Las correlaciones entre BOVA11 y SPDR S\&P500 son, en media, superiores, con un máximo de 0.723 y un mínimo de 0.042 . La menor correlación dinámica se observa entre el Ifix y el S\&P500, siendo, en media, 0.018 y tomando valores negativos durante varios períodos en 2014 .

Las correlaciones negativas observadas en algunos períodos de tiempo entre el Ifix y el SPDR S\&P50o indican un elevado potencial de diversificación.

En la segunda parte, una vez estimados los rendimientos y correlaciones condicionales con los modelos DCC y VARDCC para un período muestral de 1.015 días (10/1/2011 a 28/11/2014), realizamos las predicciones para el día siguiente. Este procedimiento de predicción se repite para los 240 días que van del 01/12/2014 al 30/10/2015. Para ello, se reestiman los dos modelos utilizando muestras rolling de 1.015 observaciones. Obtenemos, por lo tanto, 240 predicciones a un día de rendimientos y matrices de covarianzas. Con el mismo número de observaciones muestrales, mediante ventanas móviles, se obtienen las predicciones por la aproximación Plug-in.

A partir de estas predicciones, en una tercera etapa obtenemos las ponderaciones óptimas diarias en las que cada uno de los tres índices se incorpora a la cartera internacional mediante el procedimiento de optimización descrito en el epígrafe 3.1. Esto nos permite obtener los rendimientos diarios de los portafolios resultantes del 01/12/2014 al 30/10/2015. Una vez anualizados estos rendimientos, calculamos la Ratio de Sharpe de cada cartera, incluida la de referencia y, en caso de resultar negativa, la Ratio de Sharpe modificada.
La tabla 5 muestra la evaluación de la performance out-of-sample para un inversor brasileño en el índice y los dos ETF considerados, suponiendo la existencia de ventas al descubierto y sin considerar la inversión en un activo sin riesgo. En ella se incluyen rendimientos, desviaciones típicas y RS anualizados. Entre paréntesis figuran los p-valores bootstrap para los contrastes de igualdad de las RS de cada cartera con la Naïve, obtenidos utilizando la metodología de Ledoit y Wolf (2008).

La evidencia empírica basada en las predicciones de performance out-of-sample son consideradas generalmente más fiables que la evidencia basada en performance in-sample que puede ser más sensible a valores atípicos y data mining (White, 2000). Además, el análisis out-of-sample también refleja mejor la información disponible para la predicción en tiempo real (Diebold \& Rudebusch, 1991). Por esta razón se muestran sólo los resultados para el período out-of-sample.

Los resultados obtenidos indican que la cartera que permite obtener el mayor rendimiento ajustado por riesgo es la basada en las predicciones de los rendimientos y volatilidades obtenidas mediante un VAR-DCC multivariante. Su rendimiento es ligeramente superior al obtenido con la estrategia basada en la modelización DCC. En términos de reducción del riesgo de cartera, no existen diferencias importantes entre los tres portafolios, si bien todos ellos mejoran la cartera de referencia.

El mejor portafolio de diversificación internacional es el basado en el VAR-DCC, con una performance que supera ligeramente las obtenidas con las otras carteras. En todas ellas la RS difiere significativamente de la de la cartera de referencia.

En el Gráfico 6 se muestran los rendimientos acumulados de la cartera de referencia y del portafolio basado en el VARDCC en el período out-of-sample. Es preciso destacar que con este portafolio se obtienen rendimientos acumulados positivos muy superiores a los de la cartera de referencia (cartera equiponderada). 
ARTÍCULOS | REIT EN BRASIL: UNA OPORTUNIDAD DE DIVERSIFICACIÓN INTERNACIONAL

Maria Celia López-Penabad | Carmen López-Andión | Ana Iglesias-Casal | José Manuel Maside-Sanfiz

Tabla 5. Evaluación de la performance out-of-sample

\begin{tabular}{|c|c|c|c|c|}
\hline & Rendimiento & Desviación típica & Ratio Sharpe & Ratio Sharpe modificada \\
\hline Cartera Naïve & $-3,309$ & 11,985 & $-0,276$ & $-39,661$ \\
\hline \multicolumn{5}{|c|}{ Carteras óptimas } \\
\hline Plug-in & 66,809 & 7,400 & $\begin{array}{c}9,027 \\
(0,0002)\end{array}$ & 9,027 \\
\hline DCC & 66,066 & 7,362 & $\begin{array}{c}8,974 \\
(0,0002)\end{array}$ & 8,974 \\
\hline VAR-DCC & 66,572 & 7,357 & $\begin{array}{c}9,049 \\
(0,0002)\end{array}$ & 9,049 \\
\hline
\end{tabular}

Nota: Rendimientos anualizados. Entre paréntesis figuran los p-valores obtenidos en el contraste de igualdad de la RS de cada una de las carteras con la RS de la de referencia, utilizando la metodología propuesta por Ledoit y Wolf (2008).

\section{Gráfico 6. Rentabilidades acumuladas}

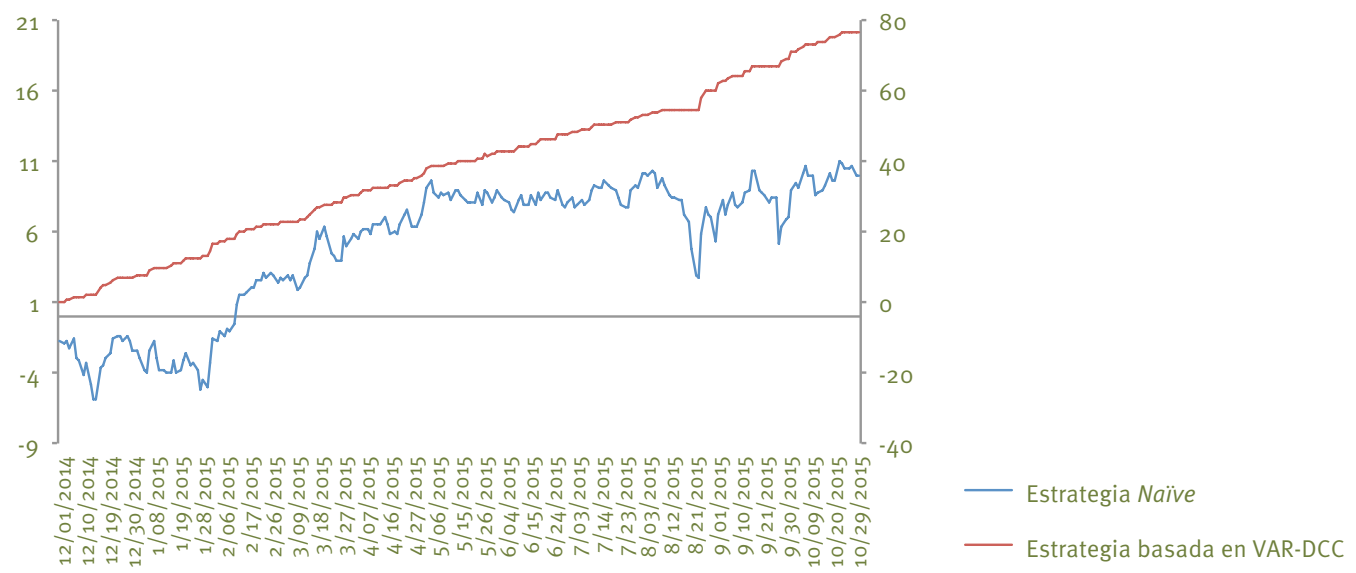

Nota: La rentabilidad acumulada de la estrategia Naïve se muestra en la escala de la izquierda, la de la estrategia VAR-DCC, en la de la derecha.

\section{ANÁLISIS DE ROBUSTEZ}

Una herramienta más flexible para capturar la dependencia entre variables a lo largo del tiempo es la función de cópula. Por ello hemos utilizado esta metodología alternativa con la finalidad de generar robustez en los resultados encontrados en la sección anterior. Una cópula es una distribución de probabilidad multivariante cuyas distribuciones marginales son uniformes en el intervalo $[0,1]$. Es decir, si las variables aleatorias U y V siguen cada una de ellas una distribución uniforme $(0,1)$, entonces la distribución conjunta del vector aleatorio $(\mathrm{U}, \mathrm{V})$ es una función cópula y se puede representar como $(\mathrm{U}, \mathrm{V}) \sim \mathrm{C}$.

Según Sklar (1959), la función de distribución conjunta de un vector aleatorio bivariante (X,Y) se puede escribir como:

$$
F(x, y)=C\left(F_{X}(x), F_{Y}(y)\right)
$$


donde $F_{X}$ y $F_{Y}$ son las distribuciones marginales de $X$ e $Y$ respectivamente y $C$ es la función cópula que describe la estructura de dependencia entre $\mathrm{X}$ e $\mathrm{Y}$.

Suponiendo que todas las funciones son diferenciables, la función de densidad conjunta viene dada por:

$$
f(x, y)=c(u, v) \cdot f_{X}(x) \cdot f_{Y}(y)
$$

donde $u=F_{x}(x), \quad v=F_{Y}(y)$ y $c(u, v)=\frac{\delta^{2} C(u, v)}{\delta u \delta v}$ es la función de densidad de la cópula.

Una característica de la cópula es que puede separar la estructura de dependencia de las distribuciones marginales y los parámetros pueden ser estimados individualmente. Por lo tanto, la cópula se puede usar para conseguir estructuras de dependencia multivariante a partir de las distribuciones marginales.

Del mismo modo que la volatilidad condicional de las series temporales cambia a lo largo del tiempo, se puede considerar que la estructura de dependencia dada por la cópula también lo hace. Existen diferentes tipos de cópulas que permiten dependencia variando en el tiempo. Las utilizadas aquí son la normal, la cópula SJC (Symmetrized Joe-Clayton), y la cópula t de Student. Todas ellas se describen con detalle en Silva, Ziegelmann y Dueker (2014).

Siguiendo a Patton (2006), el modelo de cópula gaussiana variando en el tiempo asume que el parámetro de dependencia varía en el tiempo de acuerdo con un proceso $\operatorname{ARMA}(1, q)$ como el siguiente:

$$
\rho_{t}=\Lambda\left(\gamma_{0 N}+\gamma_{1 N} \rho_{t-1}+\gamma_{2 N} \frac{1}{q} \sum_{j=1}^{q} \phi^{-1}\left(u_{t-j}\right) \phi^{-1}\left(v_{t-j}\right)\right)
$$

donde $\Lambda($.) es la transformación logística para mantener el parámetro en el intervalo $(-1,1)$ y $\Phi$ es la función de distribución acumulada de una normal bivariante. En este proceso ARMA la componente autorregresiva captura la persistencia de la dependencia y la otra componente captura los movimientos combinados de las marginales transformadas con retardos de hasta q períodos. Esta función de cópula no permite dependencia de colas a diferencia de las que se mencionan a continuación.

Para la copula t de Student variando en el tiempo la ecuación que describe la evolución del parámetro de dependencia es la siguiente:

$$
\rho_{t}=\Lambda\left(\gamma_{0 T}+\gamma_{1 T} \rho_{t-1}+\gamma_{2 T} \frac{1}{q} \sum_{j=1}^{q} T_{n}^{-1}\left(u_{t-j}\right) T_{n}^{-1}\left(v_{t-j}\right)\right)
$$

y la evolución de los grados de libertad viene dada por la expresión:

$$
n_{t}=\Lambda\left(\gamma_{3 T}+\gamma_{4 T} n_{t-1}+\gamma_{5 T} \frac{1}{q} \sum_{j=1}^{q} T_{n}^{-1}\left(u_{t-j}\right) T_{n}^{-1}\left(v_{t-j}\right)\right)
$$

siendo $T_{n}$ la función de distribución acumulada de una t de Student con $n$ grados de libertad.

En el caso de la SJC variando en el tiempo, los parámetros de dependencia de cola superior e inferior se obtienen a partir de las siguientes ecuaciones dinámicas:

$$
\tau_{t}^{U}=\Lambda\left(\gamma_{0 U}+\gamma_{1 U} \tau_{t-1}^{U}+\gamma_{2 U} \frac{1}{q} \sum_{j=1}^{q}\left|u_{t-j} v_{t-j}\right|\right)
$$




$$
\tau_{t}^{L}=\Lambda\left(\gamma_{0 L}+\gamma_{1 L} \tau_{t-1}^{L}+\gamma_{2 L} \frac{1}{q} \sum_{j=1}^{q}\left|u_{t-j} v_{t-j}\right|\right)
$$

El procedimiento de estimación se efectúa en dos etapas. En la primera etapa se estiman los parámetros de las marginales y en la segunda, a partir de éstos, se estiman los parámetros de la cópula. Para la modelización de las marginales utilizamos el mismo modelo que en la parte univariante del modelo DCC de la sección de Metodología, es decir, un modelo ARMA en la media y t-TARCH en la varianza condicional para cada serie de rendimientos, cuyos resultados se detallan en la Tabla 2. A continuación, se estiman las funciones de cópulas para capturar la dependencia entre cada uno de los posibles pares de rendimientos. En cada caso se han estimado las tres alternativas descritas anteriormente y se ha seleccionado la óptima utilizando como criterios el logaritmo de la función de verosimilitud y los estadísticos AIC y BIC. Los resultados de la estimación de las cópulas seleccionadas se muestran en la Tabla 6. Las correlaciones variando en el tiempo dadas por estas estimaciones se utilizan para la asignación de activos en la cartera como alternativa a las correlaciones dinámicas dadas por los modelos multivariantes DCC y VAR-DCC.

\section{Tabla 6. Resultados de los modelos de cópula}

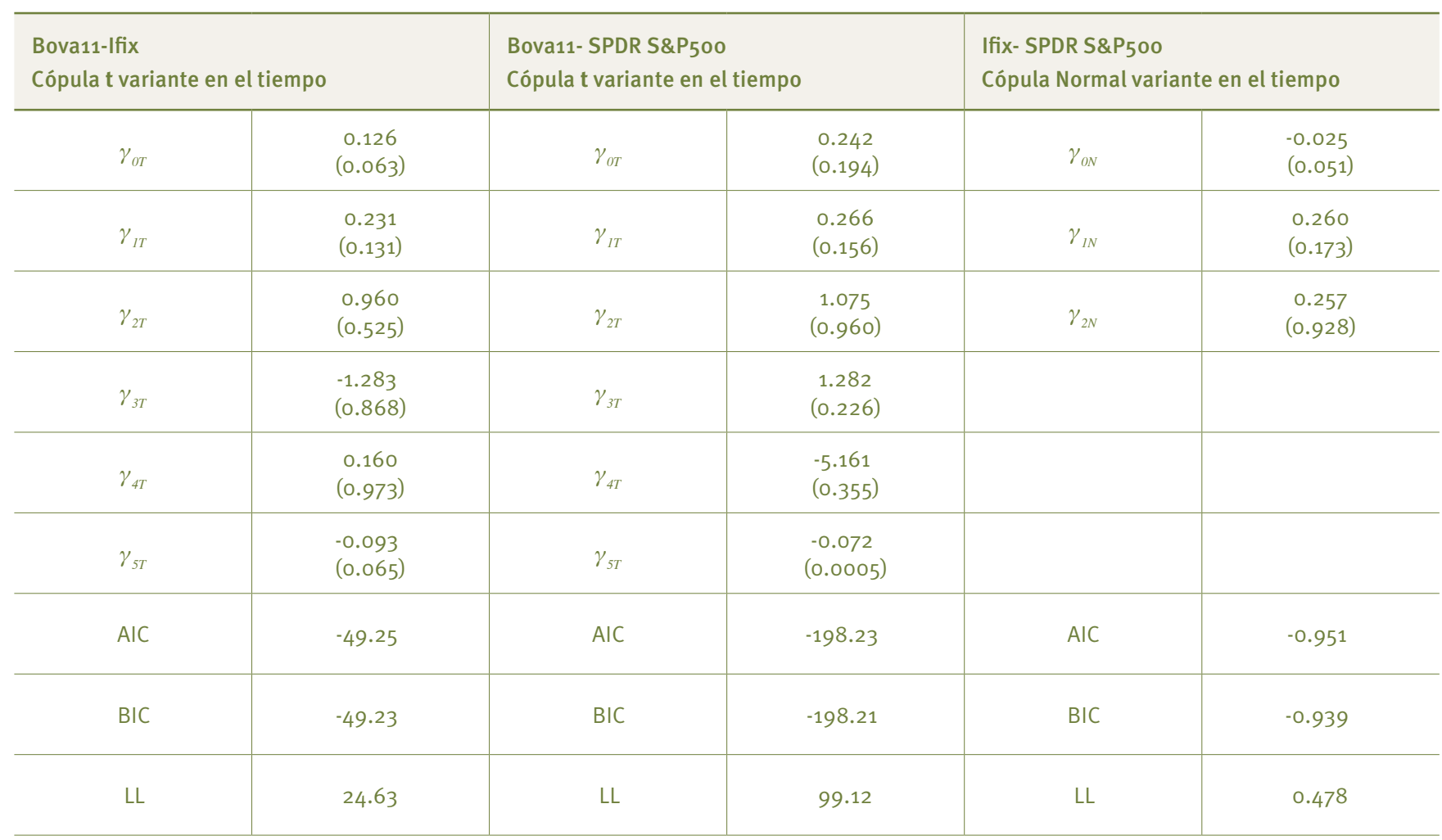

Nota: Resultados de la estimación por máxima verosimilitud. Entre paréntesis figuran los errores estándar asintóticos. LL es el logaritmo de verosimilitud de la cópula en el máximo, AlC es el criterio de información de Akaike y BIC es el criterio de información bayesiano de Schwarz.

En la Tabla 7 se recogen los resultados de rendimiento anualizado, desviación típica y RS de la cartera basada en las cópulas finalmente seleccionadas. Estos resultados son muy similares a los obtenidos con el modelo DCC y ligeramente inferiores a los del VAR-DCC y de la cartera Plug-in, lo que indica la robustez de los resultados previos.
Tabla 7. Evaluación de la performance out-of-sample de la cartera basada en los modelos de cópulas

\begin{tabular}{c|c|c}
\hline Rendimiento & Desviación típica & Ratio Sharpe \\
\hline 65.892 & 7.368 & $\begin{array}{c}8.942 \\
(0.0002)\end{array}$ \\
\hline
\end{tabular}

Nota: Rendimientos anualizados. Entre paréntesis figuran los p-valores obtenidos en el contraste de igualdad de la RS de cada una de las carteras con la RS de la de referencia, utilizando la metodología propuesta por Ledoit y Wolf (2008). 


\section{CONCLUSIONES}

La correlación entre las distintas economías mundiales está expuesta a una continua revisión debido a la creciente interacción y globalización de los mercados. La inversión inmobiliaria y la inversión en economías emergentes constituyen dos opciones con un elevado potencial diversificador que analizamos en el presente trabajo.

Nuestro objetivo consiste en analizar la performance de la inversión inmobiliaria colectiva, a través de la inversión en REIT del mercado de Brasil, en combinación con la renta variable de Brasil y de EE.UU. Para ello, construimos portafolios óptimos, combinación del índice Ifix y de los ETF BOVA11y SPDR S\&P500, con base diaria, para un inversor domiciliado en Brasil, estimando rendimientos, varianzas y covarianzas mediante modelos DCC y VAR-DCC, y analizamos su performance out-of-sample. Comparamos los resultados con el modelo tradicional Mediavarianza, o modelo Plug-in, y con la estrategia Naïve. Realizamos también pruebas de robustez de los resultados mediante la metodología de cópulas variantes en el tiempo como alternativa a los modelos multivariantes DCC y VAR-DCC.

Los resultados obtenidos indican que la cartera que permite obtener el mayor rendimiento ajustado por riesgo es la basada en las predicciones de los rendimientos y volatilidades obtenidas mediante un VAR-DCC multivariante. Su rendimiento es levemente superior al obtenido con la estrategia basada en la modelización Plug-in y DCC. En términos de diversificación del riesgo, no existen diferencias importantes entre los portafolios, aunque la Ratio de Sharpe en la aproximación VAR-DCC es ligeramente superior, si bien todas ellas mejoran la cartera de referencia (estrategia Naïve).

Estos resultados son relevantes tanto para el mundo académico como para las gestoras de carteras profesionales. La gestión de carteras está experimentando una transformación de la mano de la robótica y la alta tecnología que utiliza, entre otros, instrumentos como los ETF o los fondos indexados en búsqueda de los mejores resultados para la inversión financiera. La consideración de datos reales de rentabilidades de los REIT de Brasil, unida a la aplicación de las técnicas de análisis multivariante de predicción de rendimientos, volatilidades y correlaciones, permite aportar valiosa información sobre la inversión inmobiliaria colectiva, en un mercado emergente como el de Brasil, relativamente nuevo. Sin duda, el trabajo permite una mayor aproximación a los mercados inmobiliarios de Latinoamérica por parte de inversores individuales e inversores institucionales en busca de activos con un atractivo potencial de rentabilidad y diversificación de riesgos.

\section{REFERENCIAS}

BM\&FBovespa. (2015). Informe de mercado. Recuperado de www.bmfbovespa.com.br/en_us/services/market-data/reports/2015

Diebold, F. X., \& Rudebusch, G. (1991). Forecasting output with the composite leading index: A real-time analysis. Journal of American Statistical Association, 86, 603-610.

Engle, R. (2002). Dynamic conditional correlation: A simple class of multivariate generalized autoregressive conditional heteroskedasticity models. Journal of Business and Economics Statistics, 20(3), 339-350. doi: $10.1198 / 073500102288618487$

Infomoney. (2014). Fundos imobiliários negociados na Bovespa. Recuperado de http://www.infomoney.com.br/imoveis/fundos-imobiliarios/noticia/2617769/saiba-onde-investem-todos-fundos-imobiliarios-negociados-bovespa\#recebiveis

Israelsen, C. L. (2003). Sharpening the sharpe ratio. Financial Planning, 33, 49-51.

Israelsen, C. L. (2005). A refinement to the sharpe ratio and information ratio. Journal of Asset Management, 5, 423-427.

Kirby, C., \& Ostdiek, B. (2012, April). It's all in the timing: Simple active portfolio strategies that outperform Naïve diversification. Journal of Financial and Quantitative Analysis, 47(2), 437-467. doi: 10.1017/ So022109012000117

Ledoit, O., \& Wolf, M. (2008). Robust performance hypothesis testing with the sharpe ratio. Journal of Empirical Finance, 15, 850-859. doi:10.1016/j.jempfin.2008.03.002

Markowitz, H. (1952, March). Portfolio selection. The Journal of Finance, 7(1), 77-91. doi: 10.1111/j.1540-6261.1952.tb01525.x

Milani, B., Ceretta, P. S., \& Machado, M. E. R. (2014). Brazilian REITs performance: An analysis of higher moments and time scales influence. WSEAS Transactions on Business and Economics, 13, 193-206. doi: $10.2139 /$ ssrn. 2553852

Patton, A. J. (2006). Modelling asymmetric exchange rate dependence. International Economic Review, 47(2), 527-556. doi: 10.1111/j. 1468-2354.2006.00387.x

Sanfelici, D. (2013). Financeirização e a produção do espaço urbano no Brasil: Uma contribuição ao debate. -EURE, 39(118), 27-46. doi: 10.4067/S0250-71612013000300002

Silva, O. C., Filho, Ziegelmann, A., \& Dueker, M. J. (2014). Assessing dependence between financial market indexes using conditional time-varying copulas: Applications to value at risk (VaR). Quantitative Finance, 14(12), 2155-2170. doi: 10.1080/14697688.2012.739726

Sklar, A. (1959). Fonctions de répartition à n dimensions et leurs marges. Publications de l'Institut Statistique de l'Université de Paris, 8, 229231. doi : 10.12691/ijefm-3-2-3

White, H. (2000). A reality check for data snooping. Econometrica, 68, 1097-1126. doi: 10.1111/1468-0262.00152 